ISSN 0103-8478

\title{
Equilíbrio de cavalos da raça Quarto de Milha participantes da modalidade de três tambores por meio de proporções corporais
}

\author{
Balance of Quarter Horses participating in the \\ barrel racing modality by using body proportions
}

\author{
Ana Carolina Donofre ${ }^{\mathrm{I} *}$ José Nicolau Prospero Puoli Filho $^{\mathrm{I}}$ \\ Iuri Emmanuel de Paula Ferreira ${ }^{\mathrm{II}}$ Marcílio Dias Silveira da Mota ${ }^{\mathrm{I}}$ \\ Marcos Chiquitelli Neto ${ }^{\text {III }}$
}

RESUMO

Baseado no conceito de que cavalos equilibrados possuem simetria entre suas regiões anatômicas, o objetivo deste trabalho foi introduzir um método de avaliação do equilíbrio de equinos atletas por meio de proporções corporais. Foram mensuradas doze medidas lineares, utilizando fita métrica, em 234 cavalos da raça Quarto de Milha durante provas de três tambores. A partir dessas mensurações, construíram-se nove proporções corporais, dadas pela razão entre duas medidas específicas. Para cada proporção, considerou-se o efeito do sexo, do peso, da idade $e$ do grau de sangue; na verificação dos testes de significância, empregou-se a estatística $F$, adotando $P<0,05$. Verificou-se que as medidas lineares do comprimento da articulação escápulo-umeral à tangente vertical do processo espinhoso da quinta vértebra torácica; da distância da tangente axial do processo espinhoso da quinta vértebra torácica à quinta vertebra lombar; e da distância da tangente vertical oblíqua da margem dorsal da região femorotíbio-rotuliana à articulação intertársica foram as principais responsáveis pela desproporcionalidade dos cavalos avaliados. $O$ efeito do sexo foi significativo para quatro das nove proporções avaliadas $\left(R_{1}, R_{2}, R_{3}\right.$ e $\left.R_{4}\right)$, sendo que as fêmeas apresentaram-se mais distantes do conceito de equilibrio, quando comparadas aos machos.

Palavras-chave: conformação corporal, equinos, razões, medidas lineares.

\section{ABSTRACT}

Based on the concept that horses have balanced symmetry between its anatomical regions, the aim of this study was to introduce a method of assessing the balance of equines through body proportions. Altogether twelve linear measurements, using metric tape, were measured in 234 horses Quarter Horses for evidence of barrel racing. From these measurements were built nine body proportions, each one given by the ratio of two specific measures, and the effects of sex, weight, age and degree of blood on the balance were evaluated. To check the significance tests we used the $F$ statistic, considering $P<0.05$. It was found that the linear measurements ML2, ML3 and ML5 were mainly responsible for the disproportionality of the horses evaluated. The effect of sex was significant $(P<0.05)$ for four proportions $(R 1, R 3, R 4$ and $R 9)$ and females were more distant from the concept of balance than males.

Key words: body conformation, horses, reasons, linear measurements.

\section{INTRODUÇÃO}

Considerado o cavalo mais versátil do mundo, o Quarto de Milha tornou-se nos últimos anos uma das principais raças dentro do mercado brasileiro de equinos. Esses animais são adaptáveis as mais diversas modalidades esportivas, com merecido destaque às provas de velocidade, como a de três tambores.

Assim como todos os bons atletas, o Quarto de Milha deve apresentar uma adequada conformação corporal, equilíbrio e simetria, independente da função a que se destina (HEDGE, 2004). A conformação é definida por THOMAS (2005) pela maneira em que as partes do corpo do animal são dispostas, se relacionam e funcionam. Segundo o mesmo autor, determina como um cavalo se move e quão bem ou mal seu corpo pode suportar os efeitos da movimentação.

\footnotetext{
IFaculdade de Medicina Veterinária e Zootecnia (FMVZ), Universidade Estadual Paulista (UNESP), 18618-970, Botucatu, SP, Brasil. E-mail: acdonofre@gmail.com.*Autor para correspondência.

IILaboratório de Ciências Exatas (LCE), Escola Superior de Agricultura Luiz de Queiroz (ESALQ), Universidade de São Paulo (USP), Piracicaba, SP, Brasil.

IIIFaculdade de Engenharia de Ilha Solteira (FEI), UNESP, Ilha Solteira, SP, Brasil. 
O equilíbrio de um equino, por sua vez, está relacionado à proporcionalidade de suas partes. As proporções são as relações existentes entre as diversas regiões anatômicas do animal e o conjunto formado por elas. Geralmente, um cavalo equilibrado apresenta experiências de menor desgaste em suas articulações, é mais apto a permanecer no esporte e ter uma longa vida de serviço (HEDGE, 2004; THOMAS, 2005).

A avaliação morfométrica é muito utilizada na escolha de equinos, especialmente para atividades esportivas, visto que a forma do corpo define os limites e a capacidade dos animais em realizarem movimentos (MAWDSLEY et al., 1996; GODOI et al., 2013). Todavia, apesar da relevância do assunto, estudos sobre a conformação corporal e o equilíbrio de equinos competidores de modalidades esportivas são raramente encontrados na literatura.

LAGE (2009) menciona a inexistência de critérios para a avaliação de diversas regiões anatômicas e zootécnicas dos equinos. Por sua vez, MAWDSLEY et al. (1996) e BEEMAN (2008) argumentam que a conformação normalmente é analisada de maneira visual e consequentemente subjetiva. Desse modo, levanta-se a necessidade de novos estudos que sirvam como ferramentas para seleção zootécnica e avaliação das alterações no desempenho, de modo a compreender e corrigir os defeitos que possam prejudicar ou impedir a utilização dos equinos em suas respectivas atividades (ROSSDALE \& BUTTERFIELD, 2006). Dessa forma, este trabalho teve por objetivo utilizar a metodologia das proporções corporais para avaliação do equilíbrio de cavalos da raça Quarto de Milha participantes da modalidade de três tambores.

\section{MATERIAL E MÉTODOS}

A avaliação ocorreu no ano de 2010, durante os eventos oficiais da Associação Brasileira dos Criadores de Cavalo Quarto de Milha - ABQM (Campeonato Nacional, Congresso Brasileiro, Potro do Futuro e Copa dos Campões), realizados no município de Avaré - SP. Ao todo, 234 equinos competidores da modalidade três tambores foram estudados.

Nos dias das competições, diferentes animais eram selecionados para a mensuração de um conjunto de medidas lineares corporais. Os equinos eram identificados, pelas informações apresentadas no seu registro, quanto a classe de idade (até 4 anos, entre 4 e 5 anos, maiores que 5 anos), ao sexo (macho ou fêmea), à genética (Quarto de Milha puro ou animais meio sangue) e ao peso (em quilos).
As mensurações eram sempre realizadas no lado esquerdo dos animais, por uma pessoa treinada previamente, a qual utilizou uma fita métrica, sendo que a unidade de medida adotada foi o centímetro (cm). Durante as mensurações, os cavalos foram conduzidos para superfícies cimentadas e mantidos na mesma posição, ou seja, em pé, posicionados com os quatro membros retos ao solo. Totalizaramse doze medidas lineares (ML), sugeridas por TORRES \& JARDIM (1981) e BEEMAN (2008), que são apresentadas na figura 1 e anatomicamente descritas a seguir: ML1: comprimento cranial caudal proveniente da articulação escápulo-umeral à porção caudal do músculo glúteo; ML2: comprimento a partir da articulação escápulo-umeral à tangente vertical proveniente do processo espinhoso da quinta vértebra torácica; ML3: distância da tangente axial do processo espinhoso da quinta vértebra torácica a quinta vertebra lombar; ML4: distância a partir da tangente vertical da quinta vértebra lombar à margem inguinal ventral e tangente horizontal da margem inguinal à margem caudal do músculo glúteo; ML5: distância da tangente vertical oblíqua da margem dorsal da região femoro-tíbio-rotuliana à articulação intertársica; ML6: distância da tangente vertical a partir da tuberosidade sacral até a margem dorsal da região femoro-tíbio-rotuliana; ML7: distância da tangente vertical a partir da tuberosidade coxal até a margem dorsal da região femoro-tíbio-rotuliana; ML8: distância da tangente vertical oblíqua a partir da tuberosidade coxal até a tuberosidade isquiática; ML9: distância da tangente vertical oblíqua a partir da tuberosidade isquiática até a margem dorsal da região femoro-tíbio-rotuliana; ML10: distância do toro do carpo ao solo; ML11: distância da tangente vertical da articulação intertársica ao solo; ML12: distância da tangente vertical do processo espinhoso da quinta vertebra torácica ao solo.

Foram construídas nove proporções corporais (R), dadas pela razão entre duas medidas lineares específicas $(\mathrm{x} / \mathrm{y})$, as quais foram adotadas mediante o preconizado na literatura, para os conceitos de equilíbrio corporal (OOM \& FERREIRA, 1987; TORRES \& JARDIM, 1992; HEDGE, 2004; THOMAS, 2005). Tais proporções foram: ML3/ ML2 ( $\left.\mathrm{R}_{1}\right)$; ML3/ ML4 ( $\left.\mathrm{R}_{2}\right)$; ML4/ ML2 ( $\left.\mathrm{R}_{3}\right)$; ML8/ ML9 ( $\mathrm{R}_{4}$ ); ML8/ ML7 ( $\left.\mathrm{R}_{5}\right)$; ML9/ ML7 ( $\left.\mathrm{R}_{6}\right)$; ML6/ $\operatorname{ML} 5\left(\mathrm{R}_{7}\right)$; ML12/ ML1 $\left(\mathrm{R}_{8}\right)$; e ML10/ML11 $\left(\mathrm{R}_{9}\right)$.

Primeiramente, as variáveis quantitativas (medidas lineares e proporções corporais) foram avaliadas a partir de estatística descritiva. Em seguida, um modelo linear clássico foi ajustado com a finalidade de relacionar as razões corporais com as variáveis sexo 


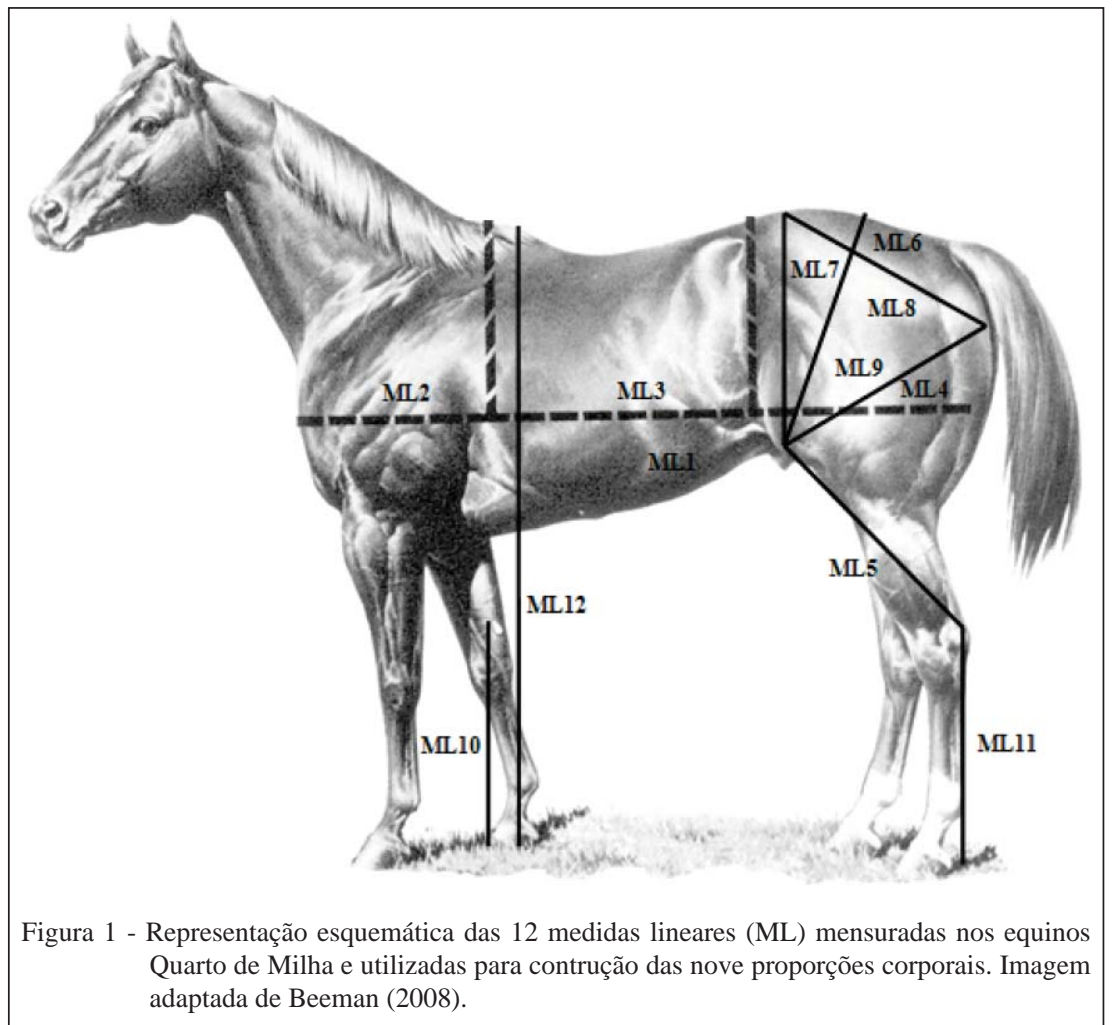

(macho ou fêmea), classe de idade (até quatro anos, entre 4 e 5 anos ou com mais de 5 anos), grau de sangue (cavalos da raça Quarto de Milha puro sangue ou meio sangue) e peso corporal. O modelo utilizado é descrito por $y_{i j k m}=\mu+s_{j}+l_{k}+\psi_{l}+\rho x_{i}+e_{i j k l m}$, em que: $y_{i j k l m}$ é o valor de determinada proporção corporal para o i-ésimo animal; $\mu$ é a média geral; $S_{j}$ é o efeito do j-ésimo sexo; $l_{k}$ é o efeito da k-ésima classe de idade; $\psi_{l}$ é efeito do l-ésimo grau de sangue; $\rho$ é o efeito de peso; $x_{i}$ é o peso do i-ésimo animal; $e_{i j k l m}$ é o erro aleatório associado a cada observação em particular.

O modelo foi ajustado aos dados para cada uma das nove proporções corporais, tidas como respostas. Em cada ajuste, o melhor conjunto de variáveis explanatórias foi selecionado a partir do Critério da Informação de Akaike (AIC). Em seguida, a qualidade do modelo ajustado para as respostas foi analisada, bem como as pressuposições clássicas para o erro aleatório foram verificadas. Por fim, testes de significância de efeitos ao nível de 5\% foram realizados a partir da estatística $\mathrm{F}$.

\section{RESULTADOS E DISCUSSÃO}

O uso das proporções corporais neste trabalho introduziu um método objetivo para avaliação do equilíbrio de equinos atletas, baseado no conceito de que cavalos equilibrados possuem igualdade no comprimento entre duas medidas lineares específicas, ou seja, são simétricos (OOM \& FERREIRA, 1987; TORRES \& JARDIM, 1992; HEDGE, 2004; THOMAS, 2005). A partir dessa teoria, adotou-se o princípio de que o valor da razão entre duas medidas proporcionais deve ser igual a um. Assim, quanto maior o distanciamento deste valor $(>1$ ou $<1$ ), maior será a desproporcionalidade corporal do animal para as medidas lineares em questão, descaracterizando o equilíbrio corporal.

De acordo com TORRES \& JARDIM (1992) e BEEMAN (2008), o comprimento cranial caudal (ML1) de um equino deve ser dividido em três terços iguais, formado pelas medidas lineares (ML) 2, 3 e 4, assim como o apresentado pelas linhas verticais da figura 1 . No entanto, na tabela 1 , observase que esta igualdade não é válida para os cavalos em estudo, visto que, em média, o comprimento da articulação escápulo-umeral à tangente vertical do processo espinhoso da quinta vértebra torácica (ML2) foi consideravelmente inferior $(37,92 \mathrm{~cm})$ quando comparado a ML3 $(61,64 \mathrm{~cm})$ e a ML4 $(51,68 \mathrm{~cm})$. Verificou-se ainda que a média da ML3 compreende a maior porção da totalidade do comprimento crânio 
Tabela 1 - Médias e medianas em centímetros e coeficientes de variação (porcentagem) das doze medidas lineares mensuradas nos 234 cavalos da raça Quarto de Milha.

\begin{tabular}{llcc}
\hline Medida linear & Média & Mediana & Cv* \\
\hline ML1 & 154,50 & 155,00 & 3,70 \\
ML2 & 37,92 & 38,00 & 7,88 \\
ML3 & 61,64 & 62,00 & 7,21 \\
ML4 & 51,68 & 52,00 & 7,07 \\
ML5 & 48,34 & 49,00 & 8,77 \\
ML6 & 58,66 & 58,00 & 6,52 \\
ML7 & 48,41 & 48,00 & 7,93 \\
ML8 & 49,35 & 50,00 & 6,34 \\
ML9 & 48,72 & 48,00 & 6,50 \\
ML10 & 55,78 & 56,00 & 4,34 \\
ML11 & 57,71 & 58,00 & 5,12 \\
ML12 & 150,50 & 150,50 & 3,06 \\
\hline
\end{tabular}

*Cv: coeficiente de variação.

caudal do corpo dos cavalos. TORRES \& JARDIM (1992) sustentam que, quando as medidas lineares $2 \mathrm{e}$ 4 são curtas, a distância da tangente axial do processo espinhoso da quinta vértebra torácica a quinta vertebra lombar (ML3) tende a ser mais comprida e defeituosa, o que torna o animal impróprio para qualquer gênero de serviço.

As medidas lineares que compõem o comprimento crânio caudal do corpo dos equinos (ML2, ML3 e ML4) refletiram nas médias das razões que as abrangem, $R_{1}, R_{2}$ e $R_{3}$ (Tabela 2), sendo que $R_{1}(1,63)$ e $R_{3}(1,37)$ revelaram com maior evidência o desequilíbrio dos animais, associado este,

Tabela 2 - Médias e desvio padrão em centímetros das proporções obtidas a partir das doze medidas lineares mensuradas nos cavalos da raça Quarto de Milha.

\begin{tabular}{llll}
\hline $\begin{array}{l}\text { Proporção } \\
\text { Corporal }\end{array}$ & $\begin{array}{l}\text { Medidas } \\
\text { lineares (ML) }\end{array}$ & Média & Sd* \\
\hline $\mathrm{R}_{1}$ & ML3/ ML2 & 1,63 & 0,152 \\
$\mathrm{R}_{2}$ & ML3/ ML4 & 1,19 & 0,115 \\
$\mathrm{R}_{3}$ & ML4/ ML2 & 1,37 & 0,132 \\
$\mathrm{R}_{4}$ & ML8/ ML9 & 1,01 & 0,085 \\
$\mathrm{R}_{5}$ & ML8/ ML7 & 1,02 & 0,085 \\
$\mathrm{R}_{6}$ & ML9/ ML7 & 1,01 & 0,095 \\
$\mathrm{R}_{7}$ & ML6/ ML5 & 1,22 & 0,173 \\
$\mathrm{R}_{8}$ & ML12/ ML1 & 0,97 & 0,039 \\
$\mathrm{R}_{9}$ & ML10/ ML11 & 0,96 & 0,040 \\
\hline
\end{tabular}

*Sd: desvio padrão principalmente ao menor comprimento médio de ML2 (37,92cm). Deve-se lembrar de que o comprimento da articulação escápulo-umeral à tangente vertical do processo espinhoso da quinta vértebra torácica, quando curto, como o observado neste estudo, pode restringir a amplitude da passada e, consequentemente, prejudicar o desempenho do animal em uma competição (INGLÊS \& VIANNA, 2004).

As medidas lineares 7, 8 e 9 compõem, segundo HEDGE (2004), o triângulo da garupa, o qual deve apresentar todos os seus lados iguais, ou seja, ser equilátero. Para o padrão racial da ABQM, a garupa deve ser longa e discretamente inclinada. Os cavalos deste estudo tiveram essas três distâncias bastante similares, o que acarretou nos resultados das razões $R_{4}(1,02), R_{5}(1,03)$ e $R_{6}(1,01)$, cujas médias ficaram bem próximas ao valor um, caracterizando um bom equilíbrio para o posterior dos cavalos Quarto de Milha.

Foi verificada a desproporcionalidade para a sétima proporção $\left(R_{7}=1,22\right)$, obtida pela razão entre a ML5 e a ML6, sendo que a média da distância da tangente vertical oblíqua da margem dorsal da região femoro-tíbio-rotuliana à articulação intertársica (ML5) foi consideravelmente menor (48,34cm). Todavia, HEDGE (2004) e a descrição do padrão racional da $\mathrm{ABQM}$, afirmam que tal desproporção pode favorecer os cavalos durante a execução da prova de três tambores, visto que uma menor ML5 é fundamental no momento da virada do tambor, pois permite voltas rápidas e paradas curtas. Este resultado levanta a hipótese de que ocorram possíveis compensações entre as medidas lineares, devido à seleção e ao treinamento no qual os cavalos competidores de provas específicas são submetidos. Entretanto, esses aspectos ainda devem ser estudados com maior cautela, visto que descaracterizam a anatomia original do cavalo e o conceito de equilíbrio corporal.

Segundo OOM \& FERREIRA (1987), cavalos bem equilibrados devem ser tão altos quanto compridos. Neste estudo, a proporção dada pela razão entre a distância da tangente vertical do processo espinhoso da quinta vertebra torácica ao solo (ML12) e o comprimento crânio caudal (ML1) obteve uma média próxima a $0,97\left(\mathrm{R}_{8}\right)$, o que representa uma boa proporcionalidade entre essas duas medidas. GODOI et a. (2013) verificaram que potros da raça Brasileiro de Hipismo também são ligeiramente mais compridos que altos, com valores dessa relação de 0,97 ; 0,97e 0,98 nas idades de 22-25; 29-32 e 36- 39 meses, respectivamente. Enquanto que as ML10 e ML11 $\left(R_{9}=0,96\right)$ denotam, também, certo 
equilíbrio entre os membros anteriores e posteriores dos animais avaliados, o que, em teoria, evita esforços desnecessários durante a movimentação (MCMANUS et al., 2008).

O modelo ajustado indicou o sexo como a variável mais influente sobre as proporções corporais, em detrimento ao peso, ao grau de sangue e à classe de idade. Da mesma forma, MISERANI et al. (2002) observaram que o efeito do sexo é uma importante fonte de variação entre as medidas lineares de equinos de outras raças.

De modo geral, as fêmeas apresentaram-se significativamente $(\mathrm{P}<0,05)$ mais distantes do conceito de equilíbrio, quando comparadas aos machos, para quatro das proporções estudadas, sendo estas R1, R3, R4 e R9. Em concordância com os resultados encontrados para o sexo, CABRAL (2004) observou que as fêmeas de cavalos da raça Mangalarga Marchador possuem uma maior distância da tangente axial do processo espinhoso da quinta vértebra torácica a quinta vertebra lombar (ML3), exposto pelo autor como comprimento do dorso-lombo, quando comparadas aos machos $(44,2 \mathrm{~cm}$ contra $47,0 \mathrm{~cm})$, bem como um menor comprimento da ML2, ou seja, da articulação escápulo-umeral à tangente vertical do processo espinhoso da quinta vértebra torácica (50,8cm contra 54,1cm). Estes mesmos resultados são refletidos nas médias das razões obtidas entre essas medidas, cujos valores de $\mathrm{R}_{1}$, para os machos, foram de 1,60 e, para as fêmeas, de 1,67.

O menor comprimento da ML2 refletiu também no valor médio da terceira proporção corporal $\left(R_{3}\right)$, em que se observou a razão de 1,33 para os machos e de 1,42 para as fêmeas. MAWDSLEY et al. (1996) também verificaram que o comprimento da ML3 foi significativamente menor em garanhões Puro-sangue Inglês, quando comparados as fêmeas, o que segundo os mesmos está relacionado ao facto de que os machos eram selecionados para provas de velocidade.As médias das razões $R_{4}$ e $R_{9}$, apesar de serem próximas em seus valores, também evidenciaram uma desproporcionalidade significativa $(\mathrm{P}<0,05)$ das fêmeas em comparação aos machos. A média de $\mathrm{R}_{4}$ para os machos foi igual a 1,00 e para as fêmeas 1,04 , enquanto que em $R_{9}$ encontraram-se valores médios de 0,96 e 0,98 para os machos e para fêmeas, respectivamente. Esse maior desequilíbrio obtido para as proporções corporais 1, 3, 4 e 9 pode estar relacionado, segundo vários autores, ao fato de existir um maior rigor, relacionado à conformação corporal, no registro genealógico de equinos machos (CABRAL, 2004; MCMANUS et al., 2005; MCMANUS et al., 2008).
As médias da classe de idade e do peso dos animais avaliados foram de 4,75 anos e 449, 46kg, respectivamente. Não houveinfluência significativa dessas variáveis sobre as proporções corporais. Todavia, para a classe de idade, em $\mathrm{R}_{1}$, $\mathrm{o}$ valor $\mathrm{p}$ foi consideravelmente baixo ( $\mathrm{P}=0,057)$, sugerindo que cavalos com mais de cinco anos sejam mais proporcionais, quanto aos comprimentos de ML2 e ML3, que os animais mais jovens.

MISERANI et al. (2002) não encontraram efeito significativo da idade em medidas de altura, perímetro e comprimento. Todavia, sabe-se que a idade pode intervir no equilíbrio dos animais, uma vez que cavalos com mais de cincos anos já apresentam o seu completo desenvolvimento, enquanto que os cavalos mais novos ainda não têm todos os seus ossos formados quando ainda possuem quatro anos de idade (MISERANI et al., 2002; MCMANUS et al., 2005).

$\mathrm{O}$ grau de sangue influenciou significativamente apenas na nona proporção corporal, dada pela distância do toro do carpo ao solo (ML10) e pela distância da tangente vertical oblíqua a partir da tuberosidade isquiática até a margem dorsal da região femoro-tíbio-rotuliana (ML9), entretanto, os valores médios foram muito próximos entre os cavalos Quarto de Milha puros $(0,97)$ e os animais meio sangue $(0,98)$. Nenhuma das variáveis foi capaz de explicar as razões $\mathrm{R}_{8}$ e $\mathrm{R}_{2}$, apesar de serem atendidas as pressuposições clássicas para o modelo ajustado. Enquanto que para as razões $R_{5}, R_{6}$ e $R_{7}$, não houve ajuste do modelo linear.

\section{CONCLUSÃO}

O uso das proporções corporais mostrouse apropriado na avaliação do equilíbrio dos animais. As medidas lineares do comprimento da articulação escápulo-umeral à tangente vertical do processo espinhoso da quinta vértebra torácica; da distância da tangente axial do processo espinhoso da quinta vértebra torácica a quinta vertebra lombar; e da distância da tangente vertical oblíqua da margem dorsal da região femoro-tíbio-rotuliana à articulação intertársica foram as principais responsáveis pela desproporcionalidade dos cavalos avaliados. O efeito do sexo foi significativo e as fêmeas apresentaram-se mais distantes do conceito de equilíbrio para quatro das nove proporções avaliadas, sendo estas $R_{1}, R_{3}, R_{4}$ e $R_{9}$.

\section{AGRADECIMENTOS}

À Fundação de Amparo a Pesquisa do Estado de São Paulo (FAPESP), pelo apoio financeiro. 


\section{COMITÊ DE ÉTICA}

Pesquisa registrada no comitê de ética da Faculdade de Medicina Veterinária e Zootecnia da Universidade Estadual Paulista (UNESP)/Botucatu, protocolo 25/2010.

\section{REFERÊNCIAS}

ASSOCIAÇÃO BRASILEIRA DOS CRIADORES DE CAVALOS QUARTO DE MILHA. Disponível em: <http://portalabqm.com. br/index.php?option=com_content\&view=article\&id=223:padraor acial\&catid=28:a-raca $>$. Acesso em: 30 out. 2013.

BEEMAN, G.M. Conformation of the horse: relationship of form to function. In: annual convention of the American association of equine practitioners, ed.54, 2008, San Diego, CA. Proceedings... San Diego: AAEP, p. 63-68, 2008.

CABRAL, G.C. et al. Avaliação morfométrica de equinos da raça Mangalarga Marchador: índices de conformação e proporções corporais. Revista Brasileira de Zootecnia, v.33, n.6, p.17981805, 2004. Disponível em: <http://www.scielo.br/pdf/rbz/ v33n6s1/a18336s1.pdf>. Acesso em: 24 fev.13. doi: 10.1590/ S1516-35982004000700018.

GODOI et al. Morfologia de potros da raça Brasileiro de Hipismo. Ciência Rural, Santa Maria, v.43, n.4, p.736-742, 2013. Disponível em: <http://www.scielo.br/scielo.php?pid=S0103$84782013000400027 \&$ script $=$ sci arttext $>$. Acesso em: 30 out. 2013. doi: 10.1590/S0103-84782013005000023.

HEDGE, J. Horse conformation, structure, soundness and performance. USA, Guilford: The Lyons, p.484, 2004.

INGLÊS, F.P.L.D.; VIANNA, S.A.B. Padrão racial comentado do cavalo Campolina. Belo Horizonte, MG: Associação Brasileira dos Criadores do Cavalo Campolina, p.28, 2004. (Boletim técnico).

LAGE, M.C.G.R. et al. Associação entre medidas lineares e angulares de equinos da raça Mangalarga Marchador. Arquivo Brasileiro de Medicina Veterinária e Zootecnia, v.61, n.4, p.968-979, 2009. Disponível em: <http://www.scielo.br/scielo.
php?script=sci_arttext\&pid=S0102-09352009000400027 $>$. Acesso em: 14 jan. 13. doi: 10.1590/S0102-09352009000400027.

MAWDSLEY, E. P. A., et al. Linear assessment of the Thoroughbred horse: an approach to conformation evaluation. Equine Veterinary Journal, v. 28, n.6, p.461-467, 1996. Disponível em: <http:// onlinelibrary.wiley.com/doi/10.1111/j.2042-3306.1996.tb01618.x/ pdf $>$. Acesso em: 30 out. 2013. doi: 10.1111/j.2042-3306.1996. tb01618.x.

MCMANUS, C.M. et al. Caracterização morfológica de equinos da raça Campeiro. Revista Brasileira de Zootecnia, v.34, n.5, p.1553-1562, 2005. Disponível em: <http://www.scielo.br/scielo. php?pid=s1516-35982005000500015\&script=sci_arttext $>$. Acesso em: 03 dez.12. doi: 10.1590/S1516-35982005000500015.

MCMANUS, C.M. et al. Body indices for the pantaneiro horse. Brazilian Journal veterinary Research Animal Science, v.45, n.5, p.362-370, 2008. Disponível em: <http://www.revistas.usp.br/ bjvras/article/view/26677/0>. Acesso em: 03 dez.12.

MISERANI, M.G. et al. Avaliação dos fatores que influem nas medidas lineares do cavalo Pantaneiro. Revista Brasileira de Zootecnia, v.31, n.1, p.335-341, 2002. Disponível em: <http:// www.scielo.br/scielo.php? script=sci_arttext\&pid=S1516$35982002000200007 \& \operatorname{lng}=$ en\&nrm=iso $>$. Acesso em 30 nov.2012. doi: 10.1590/S1516-35982002000200007.

OOM, M.M.; FERREIRA, J.C. Estudo biométrico do cavalo Alter. Revista Portuguesa de Ciências Veterinárias, v.83, n.482, p.101148, 1987.

ROSSDALE, R.D.; BUTTERFIELD, R.M. Conformation: a step along the pathway to objective assessment. Journal of Equine Veterinary, v.38, p.588-590, 2006. Disponível em: <http:// onlinelibrary.wiley.com/doi/10.1111/j.2042-3306.2006.tb01266.x/ pdf $>$. Acesso em: 22 fev. 13. doi: 0.1111/j.2042-3306.2006. tb01266.x.

THOMAS, H.S. The horse conformation handbook. USA, North Adams: Storey Publishing, p.387, 2005.

TORRES, A.D.P.; JARDIM, W.R. Criação do cavalo e de outros equinos. São Paulo: Nobel, p.654, 1992. 